\title{
A Simple and Nondestructive Technique for Estimating the Newly Grown Roots of Potted Phalaenopsis Plants
}

\author{
Keng Heng Chang ${ }^{1}$ \\ Floriculture Research Center, Taiwan Agricultural Research Institute, COA, \\ 1-10, Mayuan Village, Gukeng, Yun lin 646, Taiwan
}

Kuan Ting Chang

Department of Horticultural Science, National Chiayi University, 300 Syuefu Road, Chiayi 60004, Taiwan

\author{
Ren Shih Chung \\ Department of Agricultural Chemistry, National Taiwan University, 1 \\ Roosevelt Road, Sec. 4, Taipei 10617, Taiwan
}

Chun Tang Lu

Crop Science Division, Taiwan Agricultural Research Institute, COA, 189, Zhongzheng Road, Wufeng Dist., Taichung 413, Taiwan

Ting Fang Hsieh
Floriculture Research Center, Taiwan Agricultural Research Institute, COA,
1-10, Mayuan Village, Gukeng, Yun lin 646, Taiwan

Additional index words. root measurement, regression analysis, counting pot

\begin{abstract}
A simple and nondestructive technique, based on the line intersect principle, was developed for estimating simultaneously more traits of the newly grown roots of potted Phalaenopsis plants. Two kinds of root distribution counting pot (RDCP) were used in the present study, namely, RDCP with a large drawing grid (RDCPL) and RDCP with a small drawing grid (RDCPS). Fifty Phalaenopsis plants were randomly sampled during the cultivation period of two to eight months after transplantation for each kind of pots. The grid number of the newly grown roots of each plant was determined by both RDCPS and RDCPL, followed by measurement of other traits such as number, fresh weight, dry weight, and length of the roots. Data were subjected to regression analyses for the selection of predictive equations. The results revealed that the grid number determined by RDCPS correlated better with the traits of the newly grown roots of potted Phalaenopsis [coefficients of determination $R^{2}=0.71$ to 0.90 ; low mean squared error (MSE)] than the grid number obtained with RDCPL $\left(R^{2}=0.66\right.$ to 0.83 ; high MSE). Without tedious and timeconsuming measurements, the four linear equations obtained with RDCPS can be a simple and nondestructive estimation for predicting the number, fresh weight, dry weight, and length of the developing roots of Phalaenopsis plants grown in pots. Another 48 Phalaenopsis plants were randomly sampled during the cultivation period of two to eight months after transplantation for the validation of selected predictive equations. The accuracy of the predictive equations of the traits of newly grown roots obtained with RDCPS was different in the following order: length $>$ dry weight $>$ fresh weight $>$ number.
\end{abstract}

Roots of terrestrial plants perform many functions such as anchorage, acquisition of water and nutrients, storage, synthesis of growth regulators, propagation, and dispersal (Fitter, 2002). Therefore, assessment of the developing root is important for the proper growth of the plant (Atkinson, 2000).

Plant roots are buried in the ground wherein environmental conditions vary with respect to space and time. In the field, the

Received for publication 12 July 2012. Accepted for publication 17 Oct. 2012.

${ }^{1}$ To whom reprint requests should be addressed; e-mail changheng@tari.gov.tw. techniques based on the line intersect principle that can investigate simultaneously more traits of root growth, including the number, fresh weight, dry weight, and length.

It has been observed that roots of Phalaenopsis plants, grown in pots for commercial production, often grow into the gaps between the medium and pot wall. The condition of the newly grown roots is an indicator of not only the growth of Phalaenopsis plants, but also its marketable value (van der Knaap et al., 2005). Thus, it is standard practice to measure the newly grown roots of Phalaenopsis plants for number of roots, length, and biomass (Kubota and Yoneda, 1993; Wang, 1995, 1998; Wang and Konow, 2002; Yoneda et al., 1997). In addition, the commonly used methods for root measurement of Phalaenopsis are tedious and time-consuming. The use of a simple and nondestructive technique for the measurement of developing roots of Phalaenopsis remains unexplored. Therefore, the objective of this study was to develop a simple and nondestructive technique based on the line intersect principle for evaluating simultaneously more traits of the newly grown roots of potted Phalaenopsis plants.

\section{Materials and Methods}

Plant materials. The experiment was conducted in a greenhouse at the Floriculture Research Center (FRC) of Taiwan Agricultural Research Institute, Gukeng, Yunlin, Taiwan. One hundred white-flowered Phalaenopsis Sogo Yukidian 'V3' plants were cultivated from 2 Feb. 2010 to 2 Oct. 2010 for the selection of the predictive equations. Another 100 plants of 'V3' were cultivated from 8 July 2011 to 8 Mar. 2012 for the validation of the predictive equation. They were transplanted into $10.5-\mathrm{cm}$ clear polyethylene $(\mathrm{PE})$ pots $(0.75 \mathrm{~L})$ containing $560 \mathrm{~mL}$ of Chilean sphagnum moss (Sphagnum magellanicum Brid.) (Jenn-yioung Company, Taichung, Taiwan), one plant per pot. The sphagnum moss was soaked in water overnight before transplanting. Plants were kept in a greenhouse equipped with a pad and fan cooling system with glass panels on the roof and the sides under a natural photoperiod. Two layers of black polypropylene shade fabric were installed inside of the greenhouse. The greenhouse air temperature ranged between 22 and $30{ }^{\circ} \mathrm{C}$ and the photosynthetic photon flux was 200 to $400 \mu \mathrm{mol} \cdot \mathrm{m}^{-2} \cdot \mathrm{s}^{-1}$. Plants were fertigated with a $20 \mathrm{~N}-8.7 \mathrm{P}-$ 16.6K fertilizer (Peters Professional 20-20-20; The Scotts Co., Marysville, $\mathrm{OH}$ ) at $0.5 \mathrm{~g} \cdot \mathrm{L}^{-1}$ every 2 to 3 weeks.

Preparation and operation of the root distribution counting pot. The RDCP was designed and prepared by FRC. They were soft, transparent PE pots with the same dimensions as the growth container used in this study (diameter, $10.5 \mathrm{~cm}$; volume, $0.75 \mathrm{~L}$ ). Two kinds of RDCPs were used, namely, an RDCP with a large drawing grid (grid length $\times$ width $=2.5 \mathrm{~cm} \times 1.5 \mathrm{~cm}$ ) and an RDCP with a small drawing grid (grid length $\times$ width $=1.0 \mathrm{~cm} \times 1.0 \mathrm{~cm})($ Fig. $1 \mathrm{~A})$. The 
procedure for measuring the newly grown roots of Phalaenopsis using RDCP involved 1) replacing the original growth pot or container with RDCP (Fig. 1B-C); 2) marking the grids through which the roots have grown between the growth medium and RDCP (Fig. 1D); and 3 ) counting the number of marked grids.

Data acquisition for the selected predictive equations. Fifteen plants were randomly sampled at second and fourth months (2 Apr. 2010 and 2 June 2010) and 10 plants were randomly sampled at sixth and eighth months (2 Aug. 2010 and 2 Oct. 2010) after the transplanting. Each Phalaenopsis plant sampled was first counted for grid number by both RDCPs (RDCPS and RDCPL), and then the number, fresh weight, dry weight, and length of all new roots were recorded. The data obtained were subjected to a linear regression analysis of grid number vs. each of the root growth parameters. The predictive equations were selected based on the combination of the significance, high $R^{2}$, and low MSE of regression (Cittadini and Peri, 2006; Rouphalel et al., 2007; Wu et al., 2010).

Validation of the predictive equation. Twelve Phalaenopsis plants were randomly sampled at each second, fourth, sixth, and eighth month (8 Sept. 2011, 8 Nov. 2011, 8 Jan. 2012, and 8 Mar. 2012after the transplanting for the validation of selected predictive equations obtained with selected RDCP. Each sampled Phalaenopsis plant was measured for grid number by selected RDCP, followed by recording the number, fresh weight, dry weight, and length of the newly grown roots. The calculated root growth for these plants was also obtained using the predictive equations. Data of measured and predicted per plant were used to fit a linear regression. The slopes were tested to see if they were significantly different from the slope of the 1:1 correspondence line (Dent and Blackie, 1979). The criteria of validating the accuracy of selected predictive equations were the significance of 1:1 linear regression equation, high $R^{2}$, and no significant difference from the slope of the 1:1 correspondence line. It means the predicted values from predictive equation were very close to the measured values. Regression analyses were conducted using PC Version 9.1 of the Statistical Analyses System for windows (SAS Institute Inc., 2004).

\section{Results and Discussion}

Relationships between grid number measured by the various root distributions counting pot and traits of newly grown roots. The regression between the grid number counted on the various RDCPs and the traits of the newly grown roots was highly significantly correlated $(P<0.01)$ (Figs. 2, 3, 4 , and 5). Based on the regression analysis, RDCPS $\left(R^{2}=0.71\right.$ and MSE $\left.=10.2\right)$ showed a better relationship between grid number and the number of newly grown roots compared with RDCPL $\left(R^{2}=0.66\right.$ and MSE $=$ 11.9) (Fig. 2). A similar finding was also obtained for comparison of grid number with fresh weight, dry weight, and the length of the newly grown roots, showing that the relationship obtained with RDCPS $\left(R^{2}=0.85\right.$ to 0.90 ; low MSE) was a better fit than that obtained with RDCPL $\left(R^{2}=0.79\right.$ to 0.83 ; high MSE) (Figs. 3, 4, and 5). It showed that differences in grid size affected the correlation between measured and predicted values of the traits of newly grown roots. Based on the selective criteria of higher $R^{2}$ and lower MSE, the grid number measured by RDCPS showed better correlation with the traits of newly grown roots. The four predictive equations obtained with RDCPS could be used to evaluate the number, fresh weight, dry weight, and length of the developing roots of Phalaenopsis plants grown in pots.

Our result is similar to that reported by Marsh (1971), Newman (1966), and Tennant (1975), a method based on the line intersect
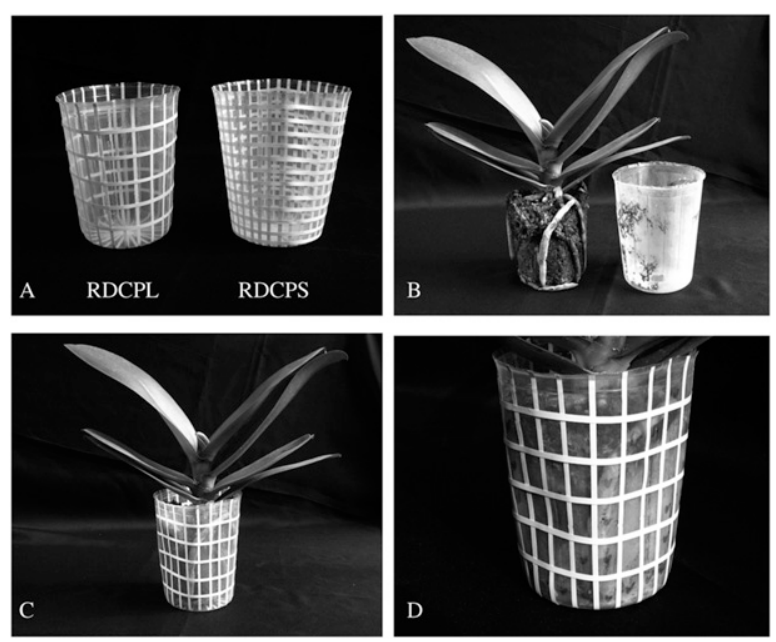

Fig. 1. (A) Two kinds of root distribution counting pots (RDCPs) with a large drawing grid (RDCPL) and a small drawing grid (RDCPS); (B-C) replacing the original growth pot by RDCP; (D) marking the grids through which the roots have grown between the growth medium and RDCP.
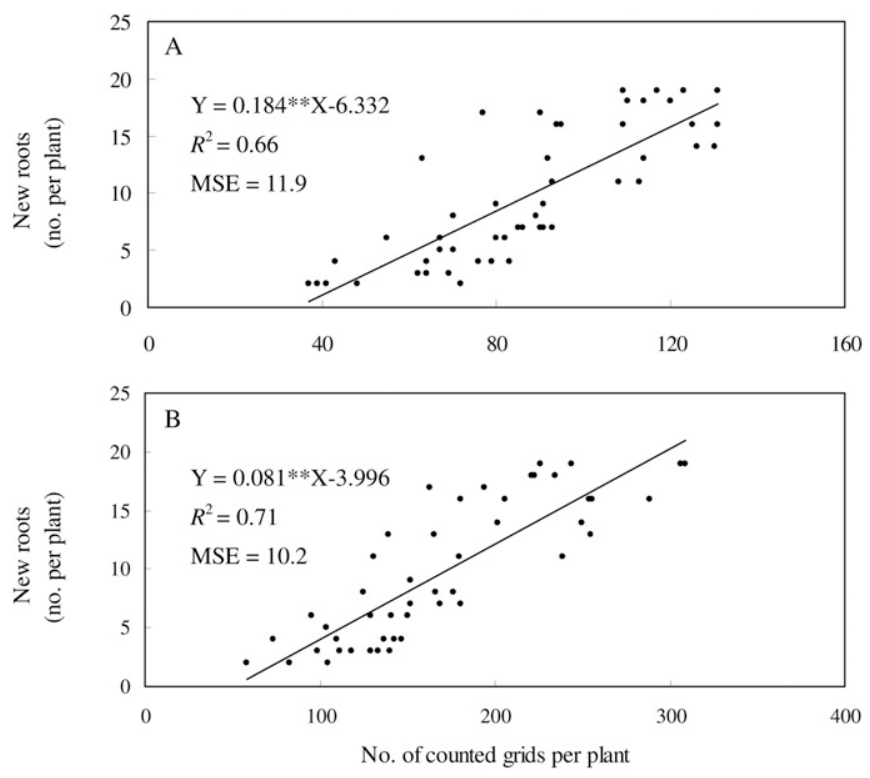

Fig. 2. Relationships between grid number measured by root distribution counting pots with (A) large and (B) small drawing grid and number of newly grown roots. $R^{2}=$ coefficients of determination; MSE = mean squared error. ${ }^{* *}$ Significant at the $1 \%$ level. 

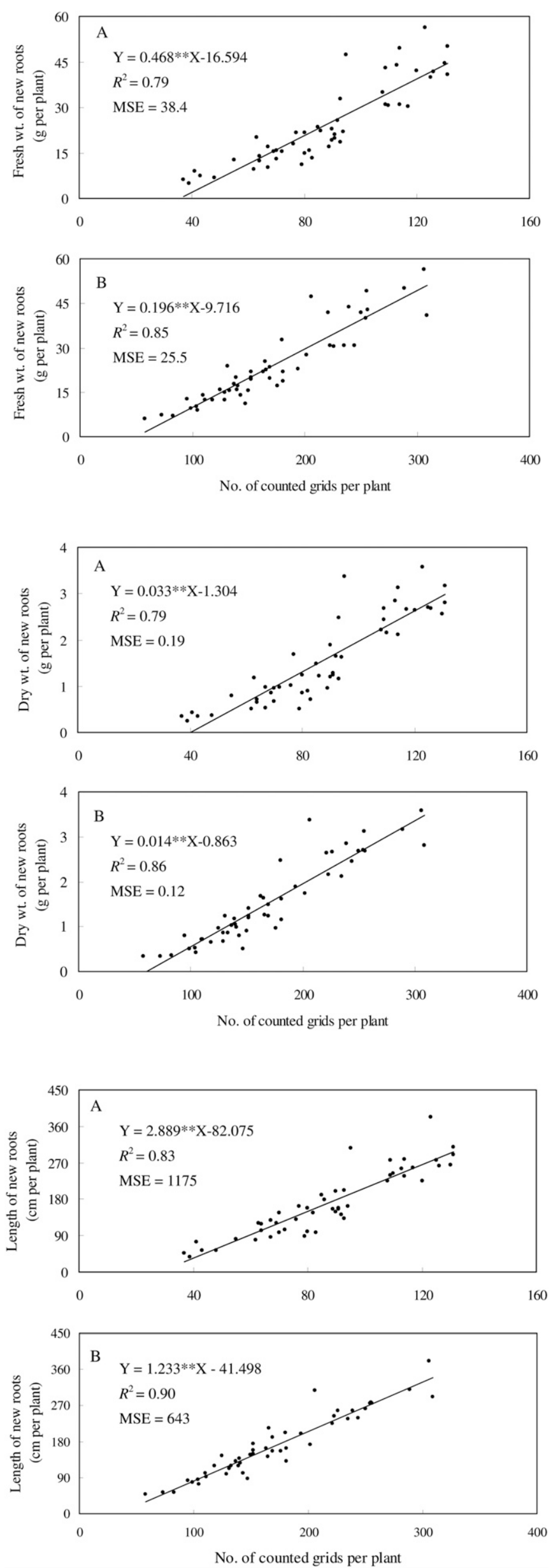

Fig. 3. Relationships between grid number measured by root distribution counting pots with (A) large and (B) small drawing grid and fresh weight of newly grown roots. $R^{2}=$ coefficients of determination; $\mathrm{MSE}=$ mean squared error. $* *$ Significant at the $1 \%$ level.
Fig. 4. Relationships between grid number measured by root distribution counting pots with (A) large and (B) small drawing grid and dry weight of newly grown roots. $R^{2}=$ coefficients of determination; MSE = mean squared error. **Significant at the $1 \%$ level.
Fig. 5. Relationships between grid number measured by root distribution counting pots with (A) large and (B) small drawing grid and length of newly grown roots. $R^{2}=$ coefficients of determination; $\mathrm{MSE}=$ mean squared error. **Significant at the $1 \%$ level. 

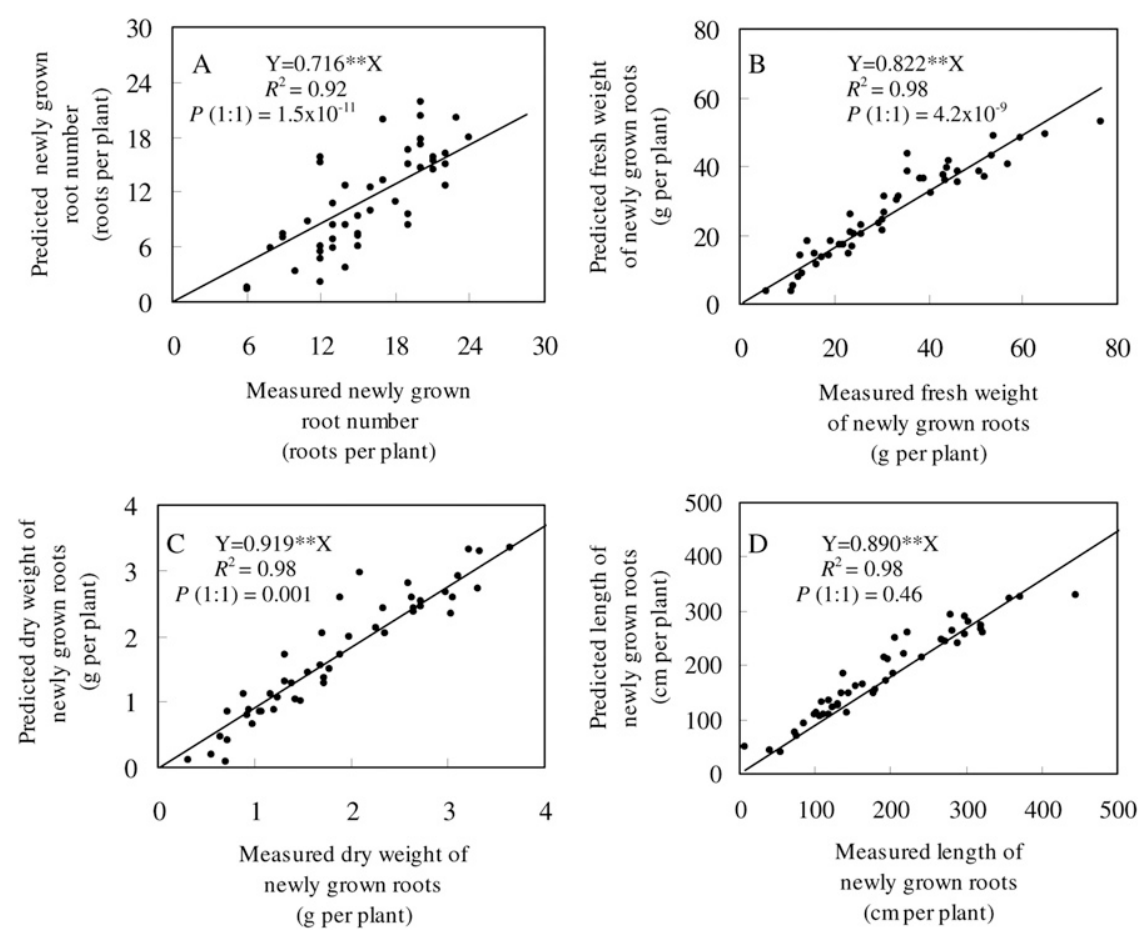

Fig. 6. Relationships between measured and predicted traits of (A) number, (B) fresh weight, (C) dry weight, and (D) length of newly grown roots. Selected predicted equations obtained with root distribution counting pots with a small drawing grid (RDCPS), "Y $=0.081 \mathrm{X}-3.996$," "Y $=0.196 \mathrm{X}-$ 9.716," "Y $=0.014 \mathrm{X}-0.863$," and "Y $=0.233 \mathrm{X}-41.498$," were used for prediction of number, fresh weight, dry weight, and length of the newly grown roots, respectively. $R^{2}=$ coefficients of determination; $\mathrm{P}(1: 1)=$ probability different from the $1: 1$ line. $* *$ Significant at the $1 \%$ level.

validate the predictive accuracy of selected equations obtained with RDCPS for evaluating the roots of Phalaenopsis plants. It showed all good fits in a linear regression between measured and predicted values for the four traits of the newly grown roots (all regression coefficients were significant and all $R^{2}>0.92$ ) (Fig. 6). According to the estimates of regression coefficient shown in Figure 6, the accuracy of the predictive equations of the traits of newly grown roots obtained with RDCPS was different in the following order: length $>$ dry weight $>$ fresh weight $>$ number. Only the regression coefficient between measured and predicted length of the predictive equations of root length was not significantly different from the $1: 1$ correspondence line $(P=0.46>0.05)$ (Fig. 6). This indicated that the predicted root length from the predictive equation obtained with RDCPS was very close to the measured root length during the cultivation of two to eight months after transplantation. The predictions of the number, fresh weight, and dry weight of the newly grown roots were slightly underestimated as a result of the fact that the regression coefficients between measured and predicted values were more than 1 . Most of the predicted values deviating from the $1: 1$ line of the root number, fresh weight, and dry weight were found to be estimated from the plants sampled at the eighth month of post-transplantation (Fig. 6). This was principally caused by the root growth of Phalaenopsis plants that often became too extensive with many of them overlapping. Thus, the predictive equations for the number, fresh weight, and dry weight of the newly grown root obtained with RDCPS were still accurate during the cultivation period of six months after transplantation.

\section{Conclusions}

The grid number determined with by RDCPS correlated better with the traits of the newly grown roots of potted Phalaenopsis than that obtained with RDCPL. The four linear predictive equations obtained with RDCPS with RDCPS can be used as a simple, time-saving, and nondestructive technique for simultaneously evaluating the number, fresh weight, dry weight, and length of the developing roots of Phalaenopsis plants grown in pots. The predictive accuracy of the equations of the traits of newly grown roots obtained with RDCPS was different in the following order: length $>$ dry weight $>$ fresh weight $>$ number. The predictive equation of root length could be satisfactorily used in the estimation of actual root length. The predictions of the number, fresh weight, and dry weight of the newly grown roots were accurate during the cultivation period of six months after transplantation, and were underestimated slightly at the eighth month of post-transplantation.

\section{Literature Cited}

Atkinson, D. 2000. Root characteristics: Why and what to measure, p. 1-32. In: Smit, A.L., A.G. Bengough, C. Engels, M. van Noordwijk, S. Pellerin, and S.C. van de Geijn (eds.). Root methods: A handbook. 1st Ed. Springer-Verlag, Berlin, Germany.
Böhm, W. 1979. Methods of studying root systems. Ecological Studies 33. Springer-Verlag, Berlin, Germany.

Cittadini, E.D. and P.L. Peri. 2006. Estimation of leaf area in sweet cherry using a non-destructive method. RIA 35:143-150.

Dent, J.B. and J. Blackie. 1979. Systems simulation in agriculture. Applied Science Publishers, London, UK.

Fitter, A. 2002. Characteristics and functions of root systems, p. 15-32. In: Waisel, Y., A. Eshel, and U. Kafkafi (eds.). Plant roots: The hidden half. 3rd Ed. Marcel Dekker, New York, NY.

Ichihashi, S. 2006. Estimation of growth, p. 152153. In: Ichihashi, S. and M. Mii (eds.). Phalaenopsia: Cultivation and production. Seibundo, Tokyo, Japan [in Japanese].

Kubota, S. and K. Yoneda. 1993. Effect of light intensity preceding day/night temperatures on the sensitivity of Phalaenopsia to flower. J. Jpn. Soc. Hort. Sci. 62:173-179 [in Japanese with English summary].

Marsh, B.a'B. 1971. Measurement of length in random arrangements of line. J. Appl. Ecol. 8:265-267.

McMichael, B.L. and H. Person. 1991. Plant roots and their environment. Elsevier, Amsterdam, The Netherlands.

Melhuish, F.M. 1968. A precise technique for measurement of roots and root distribution in soils. Ann. Bot. (Lond.) 32:15-22.

Newman, E.I. 1966. A method of estimating the total length of root in a sample. J. Appl. Ecol. 3:139-145.

Polomski, J. and N. Kuhn. 2002. Root research methods, p. 295-322. In: Waisel, Y., A. Eshel, and U. Kafkafi (eds.). Plant roots: The hidden half. 3rd Ed. Marcel Dekker, New York, NY.

Rouphalel, Y.G., G. Colla, S. Fanasca, and F. Karam. 2007. Leaf area estimation of sunflower leaves from simple linear measurements. Photosynthetica 45:306-308.

SAS Institute Inc. 2004. SAS/STAT user's guide. Version 9.1. SAS Inst. Inc., Cary, NC.

Smit, A.L., A.G. Bengough, C. Engels, M. van Noordwijk, S. Pellerin, and S.C. van de Geijn. 2000. Root methods: A handbook. 1st Ed. Springer-Verlag, Berlin, Germany.

Tennant, D. 1975. A test of a modified line intersect method of estimating root length. J. Ecol. 63:995-1001.

van der Knaap, N., M. van Herk, R. Kuijf, N. van Rosmalen, L. de Goeij, W. Gijzen, M. van der Leeden, J. van Spingelen, A. Lont, and A. van Os. 2005. Cultivation guide Phalaenopsis. 1st Ed. Anthura B.V., Bleiswijk, Holland.

Waisel, Y., A. Eshel, and U. Kafkafi. 2002. Plant roots: The hidden half. 3rd Ed. Marcel Dekker, New York, NY

Wang, Y.T. 1995. Medium and fertilization affect performance of potted Dendrobium and Phalaenopsis. HortTechnology 5:234-237.

Wang, Y.T. 1998. Impact of salinity and media on growth and flowering of a hybrid Phalaenopsis orchid. HortScience 32:247-250.

Wang, Y.T. and E.A. Konow. 2002. Fertilizer source and medium composition affect vegetative growth and mineral nutrition of a hybrid moth orchid. J. Amer. Soc. Hort. Sci. 127:442447.

Wu, H.C., L.F. Chan, M.L. Wei, and H.Y. Lu. 2010. A simple and inexpensive technique for estimating leaf surface area of muskmelon (Cucumis melo L.). J. Taiwan Agr. Res. 59: 71-77.

Yoneda, K., M. Usui, and S. Kubota. 1997. Effect of nutrient deficiency on growing and flowering of Phalaenopsis. J. Jpn. Soc. Hort. Sci. 66:141147 [in Japanese with English summary]. 